\title{
Quantitative detection of disseminated cancer cells in the greater omentum of gastric carcinoma patients with real-time RT-PCR: a comparison with peritoneal lavage cytology
}

\author{
Yasuhiro Kodera ${ }^{1}$, Hayao Nakanishi ${ }^{2}$, Seiji Ito ${ }^{1}$, Yoshitaka Yamamura ${ }^{1}$, Yukihide Kanemitsu ${ }^{1}$, \\ Yasuhiro Shimizu $^{1}$, Takashi Hirai ${ }^{1}$, Kenzo Yasui ${ }^{1}$, Tomoyuki Kato ${ }^{1}$, and Masae Tatematsu ${ }^{2}$ \\ ${ }^{1}$ Department of Gastroenterological Surgery, Aichi Cancer Center Hospital, Nagoya, Japan \\ ${ }^{2}$ Division of Oncological Pathology, Aichi Cancer Center Research Institute, Nagoya, Japan
}

\begin{abstract}
Background. Peritoneal lavage cytology is an excellent prognostic determinant but lacks sensitivity. Quantification of carcinoembryonic antigen (CEA) mRNA in peritoneal washes by real-time reverse-transcriptase polymerase chain reaction (RT-PCR) was found to be a more sensitive method to detect free cancer cells. It may be beneficial to explore, by the same method, a sample of the omentum, which is known to harbor cancer cells before the establishment of gross peritoneal metastasis.

Methods. Greater omentum and peritoneal washes were obtained from 90 gastric carcinoma patients during laparotomy. The CEA mRNA levels in these materials were quantified using a real-time RT-PCR system with hybridization probes. The significance of CEA mRNA levels in both materials, as a predictive factor of peritoneal metastasis, was explored by univariate and multivariate analyses.

Results. After a median follow-up of 718 days, 13 patients had clinical evidence of peritoneal metastasis. Under the assumption that these patients had free cancer cells in the peritoneal cavity, the sensitivity and specificity of conventional cytology for the detection of these cells were $31 \%$ and $100 \%$, respectively. The sensitivity and specificity of the CEA mRNA levels extracted from the peritoneal washing samples were $77 \%$ and $94 \%$, while those of the omentum were $46 \%$ and $90 \%$. Multivariate analysis, with the diagnosis of peritoneal metastasis as an endpoint, revealed that CEA mRNA level in the peritoneal washes was the only significant risk factor.

Conclusion. Quantitative RT-PCR of peritoneal washes remains the first choice as a tool to sensitively predict intraperitoneal recurrence in gastric carcinoma patients.
\end{abstract}

Key words Carcinoembryonic antigen $\cdot$ Recurrence $\cdot$ Peritoneal metastasis

Offprint request to: $\mathrm{Y}$. Kodera

Second Department of Surgery, Nagoya University, School of Medicine, 65 Tsurumai-cho, Showa-ku, Nagoya 466-8550, Japan

Received: November 5, 2001 / Accepted: December 25, 2001

\section{Introduction}

Gastric carcinoma remains as one of the leading causes of cancer death in Japan. Peritoneal carcinomatosis represents the most common route of tumor dissemination in patients with this disease, especially when the tumor has invaded as far as the gastric serosa [1]. Recurrence in this case is most likely caused by the presence in the abdominal cavity of free cancer cells, and these can be detected by peritoneal lavage cytology [2-5]. Conventional peritoneal lavage cytology, performed by Papanicolaou and Giemsa staining, has been acclaimed as a strong prognostic determinant, and is currently considered one of the key components in the stage classification scheme of the Japanese classification of gastric carcinoma [6]. Nevertheless, conventional cytology is reported to lack sensitivity [7], and peritoneal carcinomatosis sometimes occurs in a patient in whom this examination had been negative. Improvements in this aspect have been reported by investigators who employed immunohistochemistry with panels of antibodies $[8,9]$, in addition to the Papanicolaou staining, to enhance the sensitivity of the cytology examination. We recently applied the reverse-transcriptase polymerase chain reaction technique (RT-PCR) for the sensitive detection of micrometastases in the peritoneal cavity, using carcinoembryonic antigen (CEA) as a target gene [10], and this also has led to a significant decrease in the incidence of false-negative results [11].

Milky spots, found mainly in the greater omentum, mesocolon, and Douglas cavity, are composed of numerous macrophages and lymphocyte aggregations [12]. These have been indicated to often become sites of implantation of free cancer cells and to contribute to the establishment of peritoneal metastasis [13]. Based on this finding, it can be hypothesized that gastric carcinoma cells may be found harbored in the omentum before the peritoneal deposits become visually recognizable elsewhere. Sakakura et al. [14] used the RT- 
PCR technique to analyze samples of the omentum, and found the method useful for the prediction of peritoneal recurrence in gastric carcinoma patients. We have established a new protocol for the rapid, quantitative detection of cancer cells in the peritoneal washes, using the LightCycler system (Roche Diagnostics, Mannheim, Germany), which combines rapid-cycle PCR within glass capillaries with continuous fluorescence monitoring of the PCR products, using a hybridization probe format [15]. In the present study, we reviewed the prognostic value and clinical implications of the detection of cancer cells derived from the omentum, using this LightCycler system, and we compared the sensitivity and specificity of this method with those of detection from the peritoneal washes.

\section{Patients, materials, and methods}

\section{Peritoneal washes and the greater omentum}

At the beginning of each operation, $100 \mathrm{ml}$ of saline was introduced into the Douglas cavity and aspirated after gentle stirring. These washes were centrifuged at $1800 \mathrm{rpm}$ for $5 \mathrm{~min}$ to collect intact cells, rinsed with phosphate-bufferred saline (PBS), dissolved in Isogen RNA extraction buffer (Nippon Gene, Tokyo, Japan), and stored at $-80^{\circ} \mathrm{C}$ until use. Lavage fluid from the left subphrenic cavity was obtained in a similar manner. Part of each peritoneal wash was examined cytopathologically after conventional Papanicolaou and Giemsa staining. Part of the greater omentum was resected at laparotomy and was sent to the laboratory immediately, where it was first minced into small pieces, and then aliquots (up to $5 \mathrm{~g}$ ) were incubated in $0.125 \%$ Trypsin $/ 2 \mathrm{mM}$ ethylene diamine tetraacetic acid (EDTA) for $30 \mathrm{~min}$ at $37^{\circ} \mathrm{C}$. The cellular component harvested from the omental surface was centrifuged, washed twice with PBS, and dissolved in Isogen RNA extraction buffer for storage. Samples were taken from morphologically normal portions of the greater omentum, and the macroscopically tumor-infiltrated portion was avoided.

\section{cDNA synthesis}

Frozen samples in Isogen were thawed, and total RNA was extracted, using a guanidinium-isothiocyanatephenol-chloroform-based method. Extracted total RNA, of up to $5 \mu \mathrm{g}$, was preincubated with $50 \mathrm{ng}$ of random hexanucleotide primer (Pharmacia Biotech, Uppsala, Sweden) in $9 \mu \mathrm{l}$ of solution for $10 \mathrm{~min}$ at $70^{\circ} \mathrm{C}$. After chilling on ice, $4 \mu \mathrm{l}$ of fivefold synthesis buffer ( $250 \mathrm{mM}$ Tris- $\mathrm{HCl},[\mathrm{pH}$ 8.3], $375 \mathrm{mM} \mathrm{KCl}, 15 \mathrm{mM}$ $\mathrm{MgCl}_{2}$ ], $2 \mu \mathrm{l}$ of $100 \mathrm{mM}$ dithiothreitol, $4 \mu \mathrm{l}$ of $2.5 \mathrm{mM}$ each dNTP, and $1 \mu$ l of SuperScript II RNase $\mathrm{H}^{-}$reverse transcriptase [200 units/ $\mu$ l; Invitrogen, Carlsbad, CA, USA) were added. The reaction mixture was then incubated for $50 \mathrm{~min}$ at $42^{\circ} \mathrm{C}$ and heated at $70^{\circ} \mathrm{C}$ for $15 \mathrm{~min}$, and the resultant first-strand cDNA was used for PCR amplification with the LightCycler.

\section{Real-time RT-PCR with the LightCycler}

Real-time RT-PCR was performed by a single-step method, using hybridization probes as fluorophores, as reported previously [15]. In brief, we used a CEAspecific oligonucleotide primer pair consisting of a sense primer: 5'-AACTTCTCCTGGTCTCTCAGCT-3', and an antisense primer: 5'-GCAAATGCTTTAAGGAA GAAG-3'. The hybridization probes consisted of a donor probe (5'-TGAAATGAAGAAACTACACCA GG-FL-3'), labeled at the $3^{\prime}$ end with fluorescence, and an acceptor probe (5'-LC-CTGCTATATCAG AGCAACCCCAA-P-3'), labeled at the $5^{\prime}$ end with LightCycler-Red640 and modified at the $3^{\prime}$ end by phosphorylation to block extension. To prove the integrity of the isolated RNA, real-time RT-PCR analysis for glyceraldehyde-3-phosphate dehydrogenase (GAPDH) was carried out using specific primers (sense: 5'TGAACGGGAAGCTCACTGG-3'; antisense: 5' TCCACCACCCTGTTGCTGTA-3'). The nucleotide sequences of the hybridization probes used were as follows; donor: 5'-TCAACAGCACACCCACTCCTFL-3'; acceptor: 5'-LC-CACCTTTGACTCTGGGG CT-P-3'. All primers and probes were synthesized and purified by reverse-phase HPLC by Nihon Gene Research Laboratories (Sendai, Japan).

PCR amplification of CEA mRNA, using a LightCycler instrument (Roche Diagnostics), was carried out in $10 \mu \mathrm{l}$ of reaction mixture, consisting of a master mixture containing Taq DNA polymerase, dNTP mixture and buffer (LightCycler DNA Master hybridization probes; Roche Diagnostics), $4.0 \mathrm{mM}$ $\mathrm{MgCl}_{2}, 0.25 \mu \mathrm{M}$ of sense and antisense primer, $0.4 \mu \mathrm{M}$ of each probe, and $1 \mu \mathrm{l}$ of template cDNA, in a LightCycler capillary. Prior to amplification, $0.16 \mu \mathrm{l}$ of anti-Taq DNA polymerase antibody (TaqStart antibody; Clontech Laboratories, Palo Alto, CA, USA) was added to the reaction mixture, with incubation at room temperature for $5 \mathrm{~min}$ to block primer elongation. For CEA amplification, $95^{\circ} \mathrm{C}(90 \mathrm{~s})$ for antibody inactivation was followed by 50 rounds of amplification, at $95^{\circ} \mathrm{C}$ $(0 \mathrm{~s})$ for denaturation, $50^{\circ} \mathrm{C}(10 \mathrm{~s})$ for annealing, and $72{ }^{\circ} \mathrm{C}(10 \mathrm{~s})$ for extension, with a temperature slope of $20^{\circ} \mathrm{C}$ per s, performed in the LightCycler Instrument. Real-time PCR monitoring was achieved by measuring the fluorescent signal at the end of the annealing phase for each cycle. For GAPDH amplification, the same temperature profile was used, except for the extension step, which was done at $72^{\circ} \mathrm{C}$ for $20 \mathrm{~s}$. 
A moderately differentiated, CEA-producing adenocarcinoma cell line, derived from a liver metastasis in a colon cancer patient (COLM-2), was used for preparing external standards for CEA mRNA, with tenfold serial dilutions of cDNA ( 1 to $10^{5}$ COLM-2 cells). The current assay system RT-PCR could detect reproducibly from $10^{\circ}(=1)$ to $10^{5}$ COLM-2 colon carcinoma cells mixed with $1 \times 10^{7}$ peripheral blood leukocytes, indicating a sensitivity comparable to that of conventional nestedprimer RT-PCR, although the low copy range $\left(10^{\circ}\right.$ cell $)$ was relatively less reproducible than the high copy range $\left(10^{1}-10^{5}\right.$ cells $)$. Each run consisted of six external standards, a negative control without a template, and patient samples with unknown mRNA concentrations. Quantitation of mRNA in each sample was then performed automatically with reference to the standard curve constructed each time by the LightCycler software (Roche Diagnostics). Of the relative CEA mRNA values for the Douglas and left subphrenic cavities obtained for each patient, the higher value was used for the subsequent analyses. In a previous study, receiver operation characteristics curve analyses were performed for CEA mRNA levels and CEA/GAPDH ratios, and no difference in the area under the curve between these two parameters was observed, indicating the correction of CEA mRNA values with respect to GAPDH to be not strictly necessary [16]. In the current study, therefore, GAPDH was used only to confirm the integrity of the RNA extracted from each sample. The cutoff value for the CEA mRNA was determined as 10 , based on another preceding study [15].

\section{Patients}

Between 1998 and 2000, samples of the greater omentum and peritoneal washes were taken from 90 patients with histologically proven gastric adenocarcinomas who underwent laparotomy at Aichi Cancer Center Hospital. These patients were followed for a median of 718 days. GAPDH mRNA was detectable in all the samples collected. The depth of cancer invasion (pT category) and the number of metastatic lymph nodes ( $\mathrm{pN}$ catogory) were evaluated and classified according to the tumor-node-metastasis classification [17], except for the four lesions that were not resected. $T$ categories for these patients were decided upon surgical findings, and $\mathrm{N}$ categories were left unrecorded. There were 46 patients with $\mathrm{T} 1$ cancer (cancer confined to the mucosa or invading as far as the submucosa), 19 patients with T2 tumors (invasion beyond the submucosa but not as far as the serosa), 16 with T3 tumors (serosal invasion), and 9 with T4 tumors (invasion to adjacent tissues). The population included 5 patients with synchronous peritoneal metastasis.

\section{Surgical procedure}

After laparotomy, the abdominal cavity was thoroughly examined for tumor metastasis, and for peritoneal deposits in particular. Samples of the latter were taken whenever they were observed, and the diagnosis of cancer metastasis was histologically confirmed with frozen sections before the abdomen was closed. When potentially curative R0 resection [17] was considered possible, gastrectomy with D2 lymphadenectomy was the treatment of choice. Palliative resection, was performed and chemotherapy given at the discretion of the surgeons for the patients who were not treated with R0 resection.

\section{Postoperative surveillance of patients}

The follow-up program consisted of interim history, physical examination, and hematology and blood chemistry panels, including tests for CEA and carbohydrate antigen (CA)19-9, performed every 3 months for the first postoperative year, and every 6 months thereafter. Either abdominal ultrasonography or computerized tomography was carried out every 6 months. Peritoneal recurrence, evident on the basis of clinical symptoms, digital examination, and physical and radiological findings of bowel obstruction and ascites, was confirmed by paracentesis, laparotomy, and autopsy, performed at the discretion of the surgeon.

\section{Statistical analysis}

Comparisons of the CEA mRNA values between various $\mathrm{T}$ categories were made with the Mann-Whitney test. Survival analyses were made by Kaplan and Meier curves, with death due to gastric carcinoma as the endpoint. One patient who died of apoplexy 47 days after surgery, and another patient who died of interstitial pneumonitis 29 days after surgery were regarded as censored cases. Univariate analysis, with diagnosis of peritoneal metastasis as the endpoint, was performed to identify risk factors for the development of peritoneal carcinomatosis. For this, cancer deaths due to other types of metastasis, in the absence of clinical signs of peritoneal carcinomatosis, were regarded as censored cases. Multivariate analysis, using the Cox regression hazards model, was then performed to identify independent risk factors for peritoneal metastasis.

\section{Results}

CEA mRNA levels in the omentum and peritoneal washes according to the depth of tumor invasion

RT-PCR analysis of GAPDH mRNA confirmed that the extracted RNA was intact in all 90 samples. Mean 


\section{Peritoneal washes}

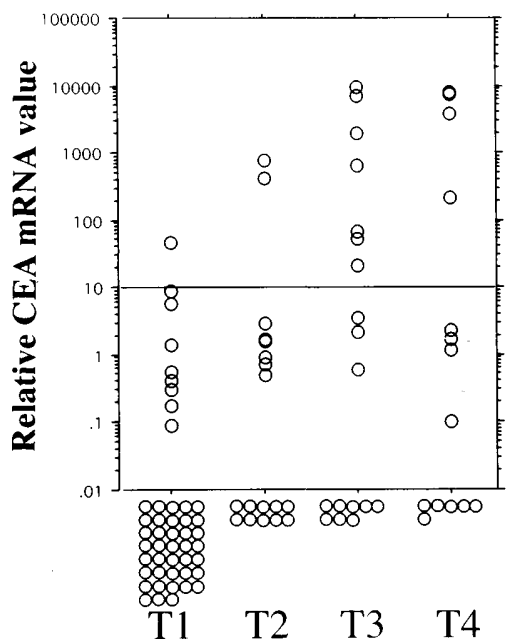

\section{Omentum}

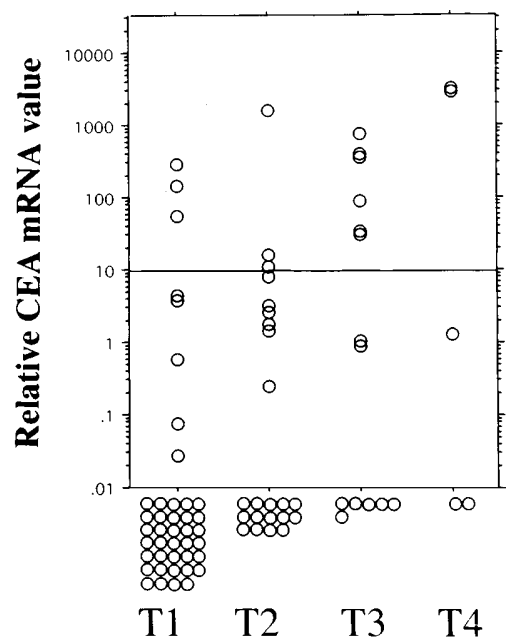

Fig. 1. Relative values of carcinoembryonic antigen (CEA) mRNA in samples of the omentum and peritoneal washes, stratified according to the pT categories by the International Union Against Cancer (UICC) stage classification. Significant differences were observed between $\mathrm{T} 1$ and T2 $(P=0.0138)$ between T1 and T3 $(P=$ $0.0051)$ for the omentum, and between T1 and T2 $(P=0.0358)$, between T1 and T3 $(P<0.0001)$, between T1 and T4 $(P<$ $0.0001)$, between T2 and T3 $(P=0.0226)$, and between $\mathrm{T} 2$ and $\mathrm{T} 4(P=0.0090)$ for the peritoneal washes

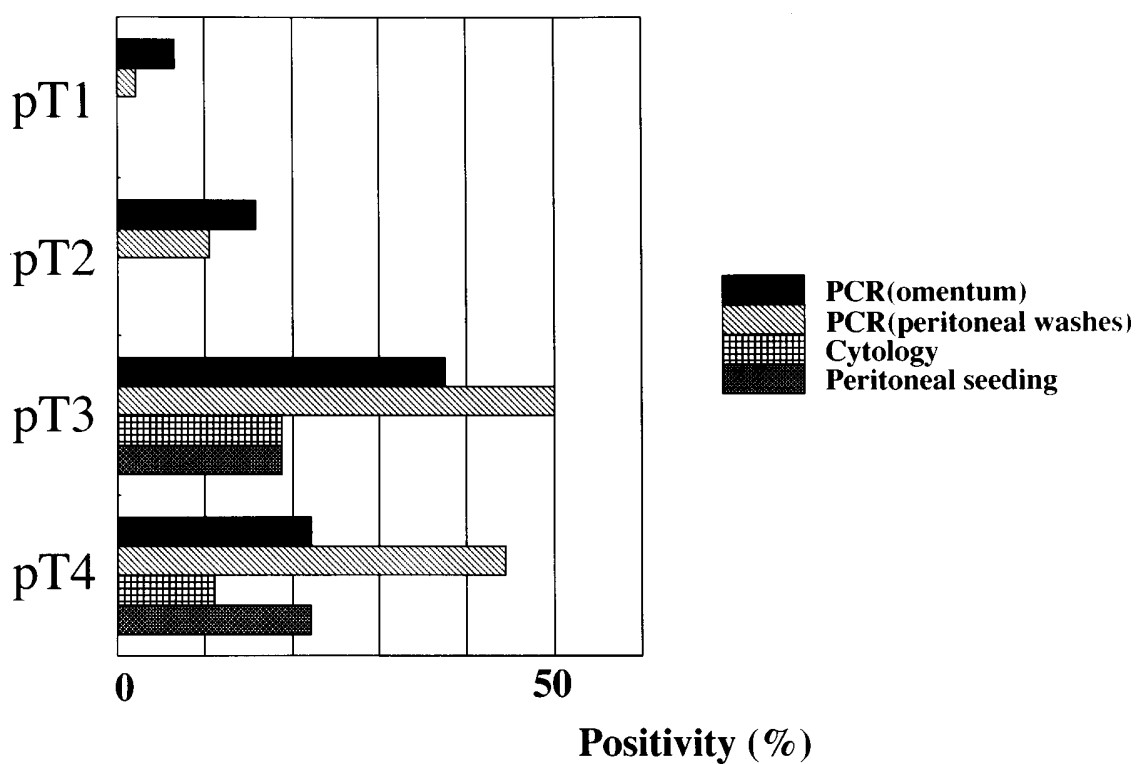

Fig. 2. The incidence of gross peritoneal metastasis, positive cytology, positive CEA mRNA for the omentum, and positive CEA mRNA for the peritoneal washes, stratified according to the pT categories by the UICC stage classification. $P C R$, Polymerase chain reaction
CEA mRNA values for the omentum varied according to the $\mathrm{T}$ category, and were $10.9 \pm 48.3$ for $\mathrm{T} 1,86.9 \pm$ 368 for T2, $105 \pm 218$ for T3, and $676 \pm 1340$ for pT4. Individual values are shown in Fig. 1. Significant differences were observed between T1 and T2 $(P=0.0138$; $\mathrm{U}$ $=305)$ and between $\mathrm{T} 1$ and $\mathrm{T} 3(P=0.0051 ; \mathrm{U}=234)$. Mean CEA mRNA values for the peritoneal washes were $1.414 \pm 7.037$ for $\mathrm{T} 1,64.6 \pm 200$ for $\mathrm{T} 2,1407 \pm$ 3013 for T3, and $2217 \pm 3450$ for pT4. Significant differences were observed between T1 and T2 $(P=0.0358$; $\mathrm{U}=325)$, between T1 and T3 $(P<0.0001 ; \mathrm{U}=149)$, between T1 and T4 $(P<0.0001 ; \mathrm{U}=46)$, between T2 and T3 $(P=0.0226 ; \mathrm{U}=87)$, and between $\mathrm{T} 2$ and $\mathrm{T} 4$ $(P=0.0090 ; \mathrm{U}=35)$.
The incidence of positive CEA mRNA levels, cytology examination, and macroscopically apparent peritoneal deposits according to the depth of tumor invasion

Figure 2 shows the incidence of positive results by conventional peritoneal lavage cytology, CEA RT-PCR with the omentum, and RT-PCR with peritoneal washing, and the incidence of concomitant peritoneal seeding at surgery, stratified according to the $\mathrm{T}$ categories. The sensitivities and specificities of each diagnostic procedure for the detection of free cancer cells were then calculated, under the assumption that the 13 patients who showed peritoneal carcinomatosis at surgery or during the follow-up had free cancer cells in the perito- 
neal cavity. Correlations between positivities for the diagnostic procedures and the incidence of peritoneal carcinomatosis are shown in Table 1. Cytology examination was rather insensitive and was positive only in three of five patients who were observed at surgery to

Table 1. Results of cytology or RT-PCR and the incidence of peritoneal carcinomatosis

\begin{tabular}{lrrr}
\hline & \multicolumn{3}{c}{$\begin{array}{c}\text { Recurrence as } \\
\text { peritoneal } \\
\text { carcinomatosis }\end{array}$} \\
\cline { 2 - 4 } & $(-)$ & $(+)$ & $P$ \\
\hline Cytology & 77 & 9 & $<0.0001$ \\
$\quad$ Negative & 0 & 4 & \\
$\quad$ Positive & 72 & 3 & $<0.0001$ \\
CEA mRNA (peritoneal washes) & 5 & 10 & \\
$\quad$ Negative & & & \\
$\quad$ Positive & 69 & 7 & 0.004 \\
CEA mRNA (omentum) & 8 & 6 & \\
$\quad$ Negative &
\end{tabular}

RT-PCR, Reverse-transcriptase polymerase chain reaction; CEA, carcinoembryonic antigen

a

Survival (\%)

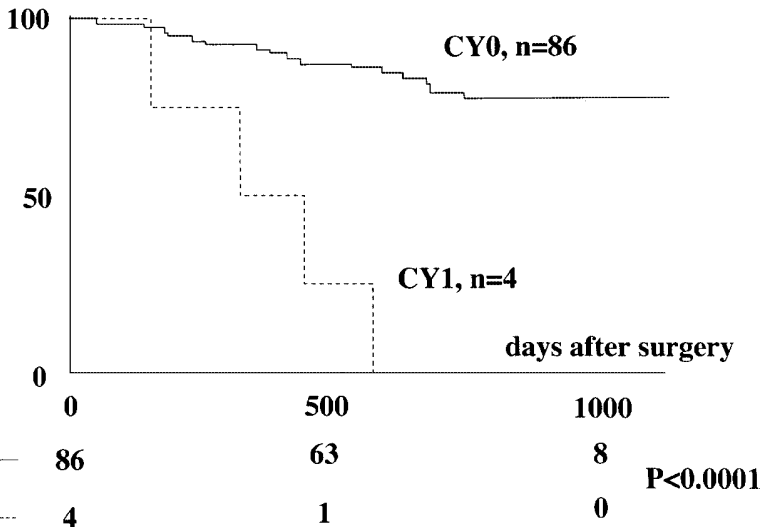

b Survival $(\%)$

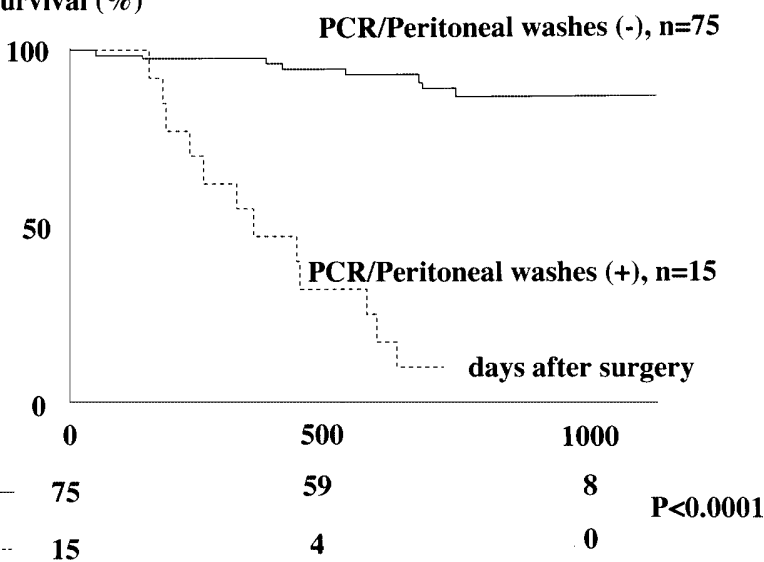

have concomitant peritoneal metastasis. There was only one other patient who had a positive cytology result. This patient, who had no peritoneal deposit at surgery, but was diagnosed after 302 days of follow-up to have developed a state of peritoneal carcinomatosis, died shortly thereafter. Consequently, the sensitivity and specificity of the conventional cytology examination were $31 \%$ and $100 \%$, respectively. At the cutoff value of 10, CEA mRNA in the omentum was positive in 14 patients, and sensitivity and specificity were $46 \%$ and $90 \%$. CEA mRNA in the peritoneal washing was positive in 15 patients, with a sensitivity and specificity of $77 \%$ and $94 \%$, respectively.

\section{Results of cytology and real-time RT-PCR and their relation to survival}

The survival curves of the 90 patients, stratified according to positivities for cytology examination and RTPCR analyses, are shown in Fig. 3. The cytology and CEA mRNA levels in the peritoneal washes were significant prognostic factors, while no significant difference in survival was observed between the patients positive and negative for the CEA mRNA in the

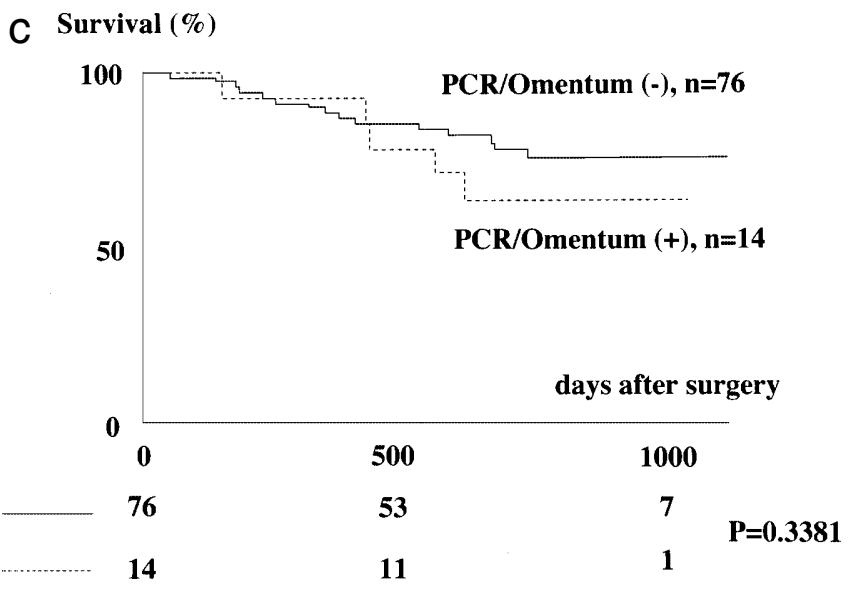

Fig. 3a-c. Survival curves of the patients who were positive and negative for a conventional peritoneal lavage cytology $(C Y)$, b CEA mRNA in the peritoneal washes, and c CEA mRNA in the omentum. Numbers at risk are shown beneath the horizontal axes. Significant differences were observed between the patients who were positive and negative for the cytology examination and those who were positive and negative for CEA mRNA in the peritoneal washes 
Table 2. Univariate analysis with the diagnosis of peritoneal metastasis as the endpoint

\begin{tabular}{|c|c|c|c|}
\hline Variables & Hazard ratio & 95\% Confidence interval & $P$ \\
\hline \multicolumn{4}{|c|}{ CEA mRNA (peritoneal washes) } \\
\hline Negative $(<10)$ & 1 & & \\
\hline Positive & 100 & $12.0-778$ & $<0.0001$ \\
\hline \multicolumn{4}{|l|}{ Cytology examination } \\
\hline Negative & 1 & & \\
\hline Positive & 25.6 & $6.83-96.7$ & $<0.0001$ \\
\hline \multicolumn{4}{|c|}{ Borrmann classification } \\
\hline Type IV & 1 & & \\
\hline Others & 7.25 & $2.35-22.4$ & 0.0006 \\
\hline \multicolumn{4}{|l|}{ Node metastasis } \\
\hline $\mathrm{pN} 0, \mathrm{pN} 1$ & 1 & & \\
\hline pN2, pN3 & 7.30 & $2.30-23.0$ & 0.0007 \\
\hline \multicolumn{4}{|l|}{ Invasion of the serosa } \\
\hline Negative & 1 & & \\
\hline Positive & 6.94 & $2.13-22.7$ & 0.0013 \\
\hline \multicolumn{4}{|c|}{ CEA mRNA (omentum) } \\
\hline Negative $(<10)$ & 1 & & \\
\hline Positive & 5.03 & $1.68-15.0$ & 0.0038 \\
\hline \multicolumn{4}{|l|}{ Histopathologic type } \\
\hline Differentiated & 1 & & \\
\hline Undifferentiated & 4.31 & $0.945-19.7$ & 0.0591 \\
\hline
\end{tabular}

Table 3. Multivariate analysis with the diagnosis of peritoneal metastasis as the endpoint

\begin{tabular}{lcc}
\hline Covariates & Hazard ratio & 95\% Confidence interval \\
\hline CEA mRNA (peritoneal wahses) & 1 & \\
$\quad$ Negative $(<10)$ & 62.5 & $4.42-848$ \\
$\quad$ Positive & 1 & \\
Cytology examination & 4.83 & $0.75-31.3$ \\
$\quad$ Negative & 1 & \\
$\quad$ Positive & 0.54 & $0.08-3.69$ \\
Invasion of the serosa & & \\
$\quad$ Negative & 1 & $0.31-8.95$ \\
$\quad$ Positive & 1.66 & \\
Node metastasis & 1 & 0.0983 \\
$\quad$ pN0, pN1 & 1.28 & $0.25-6.46$ \\
pN2, pN3 & & \\
Borrmann classification & 1 & 0.5255 \\
$\quad$ Othe IV & 1.09 & $0.25-4.83$ \\
CEA mRNA (omentum) & & \\
$\quad$ Negative (<10) & & \\
Positive & & \\
\hline
\end{tabular}

samples of the omentum. With the diagnosis of peritoneal carcinomatosis as an endpoint, positive cytology, positive CEA mRNA in the peritoneal washes and omentum, invasion of the serosa, metastasis in more than six lymph nodes $(>\mathrm{pN} 1)$, and gross finding of Borrmann type 4 cancer were significant as prognostic factors by univariate analysis (Table 2). When multivariate analysis was performed with these six covariates and the same endpoint, positive CEA mRNA in the peritoneal washes was an independent significant predictive factor for peritoneal metastasis, while positive CEA mRNA in the omentum was not (Table 3 ).

\section{Discussion}

The omentum is known to harbor cancer cells [13], and is sometimes found at laparotomy to be a focus of macroscopic cancer deposits. For this reason, it is a common practice to routinely co-resect the omentum in radical 
surgery for gastric and ovarian cancers, the cancer types with a relatively high incidence of peritoneal seeding. Detection of free cancer cells in the peritoneal washes is an established method for the assessment of risk for peritoneal carcinomatosis [6], and we have already succeeded in applying a real-time RT-PCR technique to this material [15]. In the current study, the omentum was tested as a novel and promising target to search for disseminated cancer cells that are clinically undetectable. CEA mRNA was actually detected in the RNA extracted from the omentum, and significant differences in the mean CEA mRNA levels were observed between T1 and T2 or T3. The sensitivity of the RT-PCR using the omentum was also found to be higher than that of the conventional peritoneal lavage cytology. However, several false-positive and false-negative cases existed, and prevented the CEA mRNA-positive status from becoming a significant independent factor for the prediction of peritoneal carcinomatosis, over and above the evaluation of peritoneal washes.

The sensitivity and specificity of the RT-PCR with peritoneal washing samples in this study were $77 \%$ and $94 \%$, and were comparable with the sensitivity and specificity of $80 \%$ and $94 \%$ obtained by our previous and more extensive study involving 189 patients with gastric carcinoma [16]. Undoubtedly, there is a great gain in sensitivity compared with conventional cytology examination, but a further shift in the cutoff value in order to achieve greater sensitivity results in a decrease in specificity. This is a problem inherent to the detection system involving RT-PCR, and is most probably due to the endogenous expression of CEA in noncancerous cells, notably leukocytes that may contaminate the peritoneal washing samples [18]. On the other hand, sensitivity, may never approach $100 \%$, because not all gastric carcinoma cells overexpress CEA. The poorly differentiated phenotype, in particular, is known to be associated with low expression of CEA [19]. Because the samples of peritoneal washes are taken at the beginning of surgery, it is not possible to detect free cancer cells that are scattered during surgical manipulation. This may represent another reason for the loss of sensitivity. Evaluation of peritoneal washing samples taken after gastrectomy may be necessary to overcome this problem. These samples, however, would be subject to further contamination by blood cell components and components of the gastrointestinal mucosal lining that express CEA mRNA, and may, in turn, result in an increased number of false-positives. Matrix metalloproteinase (MMP)-9 [20] and trypsinogen [21] have been reported as other potential target mRNAs, but neither was without similar problems.

GAPDH mRNA was detected in all samples of the omentum, and the current method of harvesting mRNA from these samples seems feasible. The greater inci- dence of false-positives and the consequent loss of specificity in comparison with the peritoneal washes may be attributable to the fact that the specimens of omentum contain greater amounts of non-cancerous cells that express low levels of CEA mRNA. Loss of sensitivity, on the other hand, may be due to the fact that the omentum may not harbor cancer cells diffusely, even when dissemination has occurred. This means that the positivity may, in part, depend on how much of and which part of the omentum is taken as a sample. Results from another study suggest, on the other hand, that the RT-PCR detection of CEA mRNA was more sensitive with the omentum than with the peritoneal washes, although specificity after a long-term follow-up remains to be elucidated [14]. The authors of that study [14] give no details regarding the amount and site of omentum sampling, except that "the greater omentum surrounding the tumor was resected". Sampling of regions closer to the tumor and the knowledge of where in the omentum the milky spots are densely scattered may have been clues to their success.

The present study reveals that detection with peritoneal washes is less susceptible to sampling errors than detection with omental washes, and that detection with peritoneal washes is suitable for multiinstitutional usage, which is the essential next step for evaluating the clinical role of the RT-PCR-mediated detection of micrometastasis. At the same time, a search for a more optimal target for amplification is warranted. A combination of several mRNAs that excel in specificity at the expense of sensitivity may be one of the ways of establishing a reliable detection system, until an ideal target is found.

Acknowledgments The authors thank Miss N. Yamada for expert technical assistance.

\section{References}

1. Boku T, Nakane Y, Minoura T, Takada H, Yamamura M, Hioki $\mathrm{K}$, et al. Prognostic significance of serosal invasion and free intraperitoneal cancer cells in gastric cancer. Br J Surg 1990;77:4369.

2. Koga S, Kaibara N, Iitsuka Y, Kudo H, Kimura A, Hiraoka H, et al. Prognostic significance of intraperitoneal free cancer cells in gastric cancer patients. J Cancer Res Clin Oncol 1984;108:236-8.

3. Bonenkamp JJ, Songun I, Hermans J, van de Velde CJ. Prognostic value of positive cytology findings from abdominal washings in patients with gastric cancer. Br J Surg 1996;83:672-4.

4. Kodera Y, Yamamura Y, Shimizu Y, Torii A, Hirai T, Yasui K, et al. Peritoneal washing cytology: Prognostic value of positive findings in patients with gastric carcinoma under-going a potentially curative resection. J Surg Oncol 1999;72:60-5.

5. Bando E, Yonemura Y, Takeshita Y, Taniguchi K, Yasui T, Yoshimitsu Y, et al. Intraoperative lavage for cytological examination in 1297 patients with gastric carcinoma. Am J Surg 1999; $178: 256-62$ 
6. Japanese Gastric Cancer Association. Japanese classification of gastric carcinoma. 2nd English ed. Gastric Cancer 1998;1:10-24.

7. Abe S, Yoshimura $\mathrm{H}$, Tabata $\mathrm{H}$, Tachibana $\mathrm{M}$, Monden $\mathrm{N}$, Nakamura T, et al. Curative resection of gastric cancer: limitation of peritoneal lavage cytology in predicting the outcome. J Surg Oncol 1995;59:226-9.

8. Benevolo M, Mottolese M, Cosimelli M, Tedesco M, Giannarelli $\mathrm{D}$, Vasselli S, et al. Diagnostic and prognostic value of peritoneal immunocytology in gastric cancer. J Clin Oncol 1998;16:340611.

9. Juhl H, Stritzel M, Wroblewski A, Henne-Bruns D, Kremer B, Schmiegel W, et al. Immunocytological detection of micrometastatic cells: comparative evaluation of findings in the peritoneal cavity and the bone marrow of gastric, colorectal, and pancreatic cancer patients. Int J Cancer 1994;57:330-5.

10. Nakanishi H, Kodera Y, Torii A, Hirai T, Yamamura Y, Kato T, et al. Detection of carcinoembryonic antigen-expressing free tumor cells in peritoneal washes from patients with gastric carcinoma by polymerase chain reaction. Jpn J Cancer Res 1997;88:687-92.

11. Kodera Y, Nakanishi H, Yamamura Y, Shimizu Y, Torii A, Hirai $\mathrm{T}$, et al. Prognostic value and clinical implications of disseminated cancer cells in the peritoneal cavity detected by reverse transcriptase-polymerase chain reaction and cytology. Int $\mathrm{J}$ Cancer 1998;79:429-33.

12. Dux R. Role of the greater omentum in the immunological response of mice and rats to the intraperitoneal inoculation of Ehrlich ascites tumor. Arch Immunol Ther Exp (Warsz) 1969;17: 425-32.

13. Hagiwara A, Takahashi T, Sawai K, Taniguchi H, Shimotsuma M, Okano S, et al. Milky spots as the implantation site for malignant cells in peritoneal dissemination in mice. Cancer Res 1993;53:68792 .
14. Sakakura C, Hagiwara A, Shirasu M, Yasuoka R, Fujita Y, Nakanishi M, et al. Polymerase chain reaction for detection of carcinoembryonic antigen-expressing tumor cells on milky spots of the greater omentum in gastric cancer patients: a pilot study. Int J Cancer 2001;95:286-9.

15. Nakanishi H, Kodera Y, Yamamura Y, Ito S, Kato T, Ezaki T, et al. Rapid quantitative detection of carcinoembryonic antigenexpressing free tumor cells in the peritoneal cavity of gastric cancer patients with real-time RT-PCR on the LightCycler. Int J Cancer 2000;89:411-7.

16. Kodera $\mathrm{Y}$, Nakanishi H, Ito S, Yamamura Y, Kanemitsu Y, Shimizu Y, et al. Quantitative detection of disseminated free cancer cells in peritoneal washes with real-time RT-PCR: a sensitive predictor of outcome for gastric carcinoma patients. Ann Surg 2002;235:499-506.

17. Sobin LH, Wittekind C. TNM Classification of malignant tumors, 5th ed. New York: John Wiley and Sons; 1997.

18. Goeminne J-C, Guillaume T, Symann M. Pitfalls in the detection of disseminated non-hematological tumor cells. Ann Oncol 2000; 11:785-92.

19. Kodera Y, Isobe K, Yamauchi M, Satta T, Hasegawa T, Oikawa $\mathrm{S}$, et al. Expression of carcinoembryonic antigen (CEA) and nonspecific crossreacting antigen (NCA) in gastrointestinal cancer; the correlation with degree of differentiation. Br J Cancer 1993;68:130-6.

20. Yonemura Y, Fujimura T, Ninomiya I, Kim BS, Bandou E, Sawa $\mathrm{T}$, et al. Prediction of peritoneal micrometastasis by peritoneal lavage cytology and reverse-polymerase chain reaction for matrix metalloproteinase-7 mRNA. Clin Cancer Res 2001;7:1647-53.

21. Fujimura T, Ohta T, Kitagawa H, Fushida S, Nishimura GI, Yonemura $\mathrm{Y}$, et al. Trypsinogen expression and early detection for peritoneal dissemination in gastric cancer. J Surg Oncol 1998; 69:71-5. 